\title{
ON A CLASS OF FOURTH-ORDER NONLINEAR DIFFERENCE EQUATIONS
}

\author{
MAŁGORZATA MIGDA, ANNA MUSIELAK, AND EWA SCHMEIDEL
}

Received 18 August 2003 and in revised form 22 October 2003

We consider a class of fourth-order nonlinear difference equations. The classification of nonoscillatory solutions is given. Next, we divide the set of solutions of these equations into two types: $F_{+}-$and $F_{-}$-solutions. Relations between these types of solutions and their nonoscillatory behavior are obtained. Necessary and sufficient conditions are obtained for the difference equation to admit the existence of nonoscillatory solutions with special asymptotic properties.

\section{Introduction}

Consider the difference equation

$$
\Delta\left(a_{n} \Delta\left(b_{n} \Delta\left(c_{n} \Delta y_{n}\right)\right)\right)+f\left(n, y_{n}\right)=0, \quad n \in \mathbb{N},
$$

where $\mathbb{N}=\{0,1,2, \ldots\}, \Delta$ is the forward difference operator defined by $\Delta y_{n}=y_{n+1}-y_{n}$, and $\left(a_{n}\right),\left(b_{n}\right)$, and $\left(c_{n}\right)$ are sequences of positive real numbers. Function $f: \mathbb{N} \times \mathbb{R} \rightarrow \mathbb{R}$. By a solution of (1.1) we mean a sequence $\left(y_{n}\right)$ which satisfies (1.1) for $n$ sufficiently large. We consider only such solutions which are nontrivial for all large $n$. A solution of (1.1) is called nonoscillatory if it is eventually positive or eventually negative. Otherwise it is called oscillatory.

In the last few years there has been an increasing interest in the study of oscillatory and asymptotic behavior of solutions of difference equations. Compared to second-order difference equations, the study of higher-order equations, and in particular fourth-order equations (see, e.g., $[1,2,3,4,5,6,7,8,9,10,11,12,13,14]$ ), has received considerably less attention. An important special case of fourth-order difference equations is the discrete version of the Schrödinger equation.

The purpose of this paper is to establish some necessary and sufficient conditions for the existence of solutions of (1.1) with special asymptotic properties.

Throughout the rest of our investigations, one or several of the following assumptions will be imposed: 
24 On a class of fourth-order nonlinear difference equations

(H1) $\sum_{i=1}^{\infty}\left(1 / a_{i}\right)=\sum_{i=1}^{\infty}\left(1 / b_{i}\right)=\sum_{i=1}^{\infty}\left(1 / c_{i}\right)=\infty$;

(H2) $y f(n, y)>0$ for all $y \neq 0$ and $n \in \mathbb{N}$;

(H3) the function $f(n, y)$ is continuous on $\mathbb{R}$ for each fixed $n \in \mathbb{N}$.

In [14] we can find the following existence theorem (some modification of Schauder's theorem) which will be used in this paper.

Lemma 1.1. Suppose $\Omega$ is a Banach space and $K$ is a closed, bounded, and convex subset. Suppose $T$ is a continuous mapping such that $T(K)$ is contained in $K$, and suppose that $T(K)$ is uniformly Cauchy. Then $T$ has a fixed point in $K$.

\section{Main results: existence of nonoscillatory solutions}

In this section, we obtain necessary and sufficient conditions for the existence of nonoscillatory solutions of (1.1) with certain asymptotic properties. We start with the following Lemma.

Lemma 2.1. Assume that (H1) and (H2) hold. Let $\left(y_{n}\right)$ be an eventually positive solution of (1.1). Then exactly one of the following statements holds for all sufficiently large n:

(i) $y_{n}>0, \Delta y_{n}>0, \Delta\left(c_{n} \Delta y_{n}\right)>0$, and $\Delta\left(b_{n} \Delta\left(c_{n} \Delta y_{n}\right)\right)>0$;

(ii) $y_{n}>0, \Delta y_{n}>0, \Delta\left(c_{n} \Delta y_{n}\right)<0$, and $\Delta\left(b_{n} \Delta\left(c_{n} \Delta y_{n}\right)\right)>0$.

Proof. Let $\left(y_{n}\right)$ be an eventually positive solution of (1.1). Then, by assumption (H2), $\left(\Delta\left(a_{n} \Delta\left(b_{n} \Delta\left(c_{n} \Delta y_{n}\right)\right)\right)\right)$ is eventually negative. Therefore, it is easy to see that the sequences $\left(a_{n} \Delta\left(b_{n} \Delta\left(c_{n} \Delta y_{n}\right)\right)\right),\left(b_{n} \Delta\left(c_{n} \Delta y_{n}\right)\right)$, and $\left(c_{n} \Delta y_{n}\right)$ are all monotone and of one sign, say for $n \geq n_{1}$.

Suppose that $a_{n_{2}} \Delta\left(b_{n_{2}} \Delta\left(c_{n_{2}} \Delta y_{n_{2}}\right)\right)=-c_{1}<0$ for some $n_{2} \geq n_{1}$. Hence,

$$
a_{n} \Delta\left(b_{n} \Delta\left(c_{n} \Delta y_{n}\right)\right) \leq-c_{1} \text { for } n \geq n_{2},
$$

then

$$
\Delta\left(b_{n} \Delta\left(c_{n} \Delta y_{n}\right)\right) \leq-\frac{c_{1}}{a_{n}} .
$$

Summing both sides of the last inequality from $n_{2}$ to $n-1$, we have

$$
b_{n} \Delta\left(c_{n} \Delta y_{n}\right)-b_{n_{2}} \Delta\left(c_{n_{2}} \Delta y_{n_{2}}\right) \leq-\sum_{i=n_{2}}^{n-1} \frac{c_{1}}{a_{i}} .
$$

Then $b_{n} \Delta\left(c_{n} \Delta y_{n}\right) \leq-\sum_{i=n_{2}}^{n-1}\left(c_{1} / a_{i}\right)$, which tends to $-\infty$ as $n \rightarrow \infty$.

Then there exists $c_{2}>0$ and $n_{3} \geq n_{2}$ such that

$$
b_{n} \Delta\left(c_{n} \Delta y_{n}\right) \leq-c_{2}, \quad \text { for } n \geq n_{3} .
$$

So,

$$
\Delta\left(c_{n} \Delta y_{n}\right) \leq-\frac{c_{2}}{b_{n}}
$$


Summing both sides of the last inequality form $n_{3}$ to $n-1$, we obtain

$$
c_{n} \Delta y_{n}-c_{n_{3}} \Delta y_{n_{3}} \leq-\sum_{i=n_{3}}^{n-1} \frac{c_{2}}{b_{i}},
$$

which tends to $-\infty$, as $n \rightarrow \infty$.

Then there exists $c_{3}>0$ and $n_{4} \geq n_{3}$ such that $\left(c_{n} \Delta y_{n}\right) \leq-c_{3}$, for $n \geq n_{4}$. Hence, $\Delta y_{n} \leq-c_{3} / c_{n}$. A final summation yields $y_{n}-y_{n_{3}} \leq-\sum_{i=n_{4}}^{n-1}\left(1 / c_{i}\right) \rightarrow-\infty$, which implies $\lim _{n \rightarrow \infty} y_{n}=-\infty$. This contradiction implies $a_{n} \Delta\left(b_{n} \Delta\left(c_{n} \Delta y_{n}\right)\right)>0$ eventually.

Next, assume that there exists $n_{5} \in \mathbb{N}$ such that $b_{n} \Delta\left(c_{n} \Delta y_{n}\right)<0$, for $n \geq n_{5}$, then $\left(c_{n} \Delta y_{n}\right)$ must be eventually positive for otherwise we are again led to conclude that $c_{n} \Delta y_{n}$ $-c_{n_{3}} \Delta y_{n_{3}} \leq-\sum_{i=n_{3}}^{n-1}\left(c_{2} / b_{i}\right), \lim _{n \rightarrow \infty} y_{n}=-\infty$. Thus, case (ii) is verified.

Next, suppose that $b_{n} \Delta\left(c_{n} \Delta y_{n}\right)>0$ for all $n \geq n_{1}$. Then $b_{n} \Delta\left(c_{n} \Delta y_{n}\right)>b_{n_{1}} \Delta\left(c_{n_{1}} \Delta y_{n_{1}}\right)=$ $c_{4}>0$.

Divide the above inequality by $b_{n}$ and sum from $n_{1}$ to $n-1$ to get

$$
c_{n} \Delta y_{n}-c_{n_{1}} \Delta y_{n_{1}}>c_{4} \sum_{i=n_{1}}^{n-1} \frac{1}{b_{i}} \longrightarrow \infty,
$$

as $n \rightarrow \infty$. Hence, $\left(\Delta y_{n}\right)$ is eventually positive.

Now we introduce an operator which divides the set of solutions of a special case of (1.1) into two disjoint subsets. We will prove that, for nonoscillatory solution, the first of them equals type (ii) solution and the second equals type (i) solution. We assume that $c_{n}=a_{n+1}$. Hence (1.1) takes the form

$$
\Delta\left(a_{n} \Delta\left(b_{n} \Delta\left(a_{n+1} \Delta y_{n}\right)\right)\right)=-f\left(n, y_{n}\right) .
$$

We introduce an operator as follows:

$$
F_{n}=x_{n-1}\left(a_{n} \Delta\left(b_{n} \Delta\left(a_{n+1} \Delta x_{n}\right)\right)\right)-\left(a_{n} \Delta x_{n-1}\right)\left(b_{n} \Delta\left(a_{n+1} \Delta x_{n}\right)\right) .
$$

Hence

$$
\Delta F_{n}=x_{n} \Delta\left(a_{n} \Delta\left(b_{n} \Delta a_{n+1} \Delta x_{n}\right)\right)-b_{n+1} \Delta\left(a_{n} \Delta x_{n-1}\right) \Delta\left(a_{n+2} \Delta x_{n+1}\right) .
$$

It is clear, by $(\mathrm{H} 2)$, that the operator $F_{n}$ is nonincreasing for every nonoscillatory solution $\left(y_{n}\right)$ of (2.8).

If $F_{n} \geq 0$ for all $n \in N$, then a solution $\left(y_{n}\right)$ of (2.8) is called an $F_{+}$-solution. If $F_{n}<0$ for some $n$, then $\left(y_{n}\right)$ is called an $F_{-}$-solution.

The operator $F$ divides the set of solutions of (2.8) into two disjoint subsets: $F_{+}-$and F--solutions.

Theorem 2.2. Assume that $\left(b_{n}\right)$ is a bounded sequence. Let $y$ be an $F_{+}$-solution of (2.8), then

$$
\begin{gathered}
\sum_{n=1}^{\infty} b_{n+1} \Delta\left(a_{n} \Delta x_{n-1}\right) \Delta\left(a_{n+2} \Delta x_{n+1}\right)<\infty, \\
\lim _{n \rightarrow \infty} b_{n} \Delta\left(a_{n+1} \Delta y_{n}\right)=0 .
\end{gathered}
$$


Proof. Let $\left(y_{n}\right)$ be an $F_{+}$-solution of (2.8). Then, from (2.8), we obtain

$$
\Delta F_{k}=-y_{k} f\left(k, y_{k}\right)-b_{k+1} \Delta\left(a_{k} \Delta y_{k-1}\right) \Delta\left(a_{k+2} \Delta y_{k+1}\right) \text {. }
$$

By summation, we obtain

$$
F_{n}=F_{1}-\sum_{k=1}^{n-1} y_{k} f\left(k, y_{k}\right)-\sum_{k=1}^{n-1} b_{k+1} \Delta\left(a_{k} \Delta y_{k-1}\right) \Delta\left(a_{k+2} \Delta y_{k+1}\right) .
$$

Since $F_{n} \geq 0$, we have

$$
\sum_{k=1}^{n-1} b_{k+1} \Delta\left(a_{k} \Delta y_{k-1}\right) \Delta\left(a_{k+2} \Delta y_{k+1}\right) \leq F_{1} \text {. }
$$

Therefore $\sum_{k=1}^{n-1} b_{k+1} \Delta\left(a_{k} \Delta y_{k-1}\right) \Delta\left(a_{k+2} \Delta y_{k+1}\right)<\infty$.

Because $\left(b_{n}\right)$ is a bounded sequence, then $\left(1 / b_{n}\right)$ is bounded away from zero. Hence, from (2.11) and the equality

$$
b_{n+1} \Delta\left(a_{n} \Delta y_{n-1}\right) \Delta\left(a_{n+2} \Delta y_{n+1}\right)=\frac{1}{b_{n-1}}\left(b_{n-1} \Delta\left(a_{n} \Delta y_{n-1}\right)\right)\left(b_{n+1} \Delta\left(a_{n+2} \Delta y_{n+1}\right)\right) \text {, }
$$

we obtain

$$
\lim _{n \rightarrow \infty} b_{n-1} \Delta\left(a_{n} \Delta y_{n-1}\right)=0,
$$

then

$$
\lim _{n \rightarrow \infty} b_{n} \Delta\left(a_{n+1} \Delta y_{n}\right)=0
$$

Theorem 2.3. Assume that $\left(b_{n}\right)$ is a bounded sequence. Every nonoscillatory solution $\left(y_{n}\right)$ of (2.8) is an $F_{+}$-solution if and only if $\left(y_{n}\right)$ is type (ii) solution.

Proof. We prove this theorem for an eventually positive solution.

Let $\left(y_{n}\right)$ be an eventually positive $F_{+}$-solution. Suppose for the sake of contradiction that it is type (i) solution.

Then from $\Delta\left(b_{n} \Delta\left(a_{n+1} \Delta y_{n}\right)\right)>0$, we get $b_{n} \Delta\left(a_{n+1} \Delta y_{n}\right)>b_{M} \Delta\left(a_{M+1} \Delta y_{M}\right)>0$ for sufficiently large $M$ and $n>M$.

This inequality contradicts condition (2.12) of Theorem 2.2. So, $\left(y_{n}\right)$ is type (ii) solution.

Let $\left(y_{n}\right)$ be type (ii) solution. We will show the positivity of the operator $F$ on the whole sequence. Choose $m$ sufficiently large. Then, from the definition of type (ii) solution, we have $F_{n}>0$ for $n \geq m$. Because the operator $F$ is nonincreasing, hence $F_{j} \geq F_{m}>0$ for all $j<m$. Since $m$ was taken arbitrary, then $F_{n}>0$ for all $n \in \mathbb{N}$. So, $\left(y_{n}\right)$ is an $F_{+}-$ solution.

Remark 2.4. Assume that $\left(b_{n}\right)$ is a bounded sequence. Then every nonoscillatory solution of (2.8) is an $F_{-}$-solution if and only if $\left(y_{n}\right)$ is type (i) solution. 
Now we turn our attention to (1.1). We introduce the notation

$$
P_{n, N}=\sum_{k=N+2}^{n} \frac{1}{a_{k}} \sum_{j=N+2}^{k} \frac{1}{b_{j}} \sum_{i=N+2}^{j} \frac{1}{c_{i}}, \quad Q_{n, N}=\sum_{k=N}^{n-1} \frac{1}{c_{k}} \sum_{j=N}^{k-1} \frac{1}{b_{j}} \sum_{i=N}^{j-1} \frac{1}{a_{i}} .
$$

Note that $Q_{n, N}$ can be written in the form

$$
Q_{n, N}=\sum_{i=N}^{n-1} \frac{1}{a_{i}} \sum_{j=i+1}^{n-1} \frac{1}{b_{j}} \sum_{k=j+1}^{n-1} \frac{1}{c_{k}}
$$

Lemma 2.5. Assume conditions ( $\mathrm{H} 1)$ and $(\mathrm{H} 2)$ hold. If $\left(y_{n}\right)$ is an eventually positive solution of (1.1), then there exist positive constants $C_{1}$ and $C_{2}$ and integer $N$ such that

$$
C_{1} \leq y_{n} \leq C_{2} Q_{n, N}
$$

for $n \geq N+3$.

Proof. Let $\left(y_{n}\right)$ be an eventually positive solution of (1.1). Then $y_{n}>0$ for large $n$. From Lemma 1.1, $\Delta y_{n}>0$ eventually, and so $y_{n} \geq C_{1}>0$.

Now we prove the right-hand side of (2.21). From (1.1) and (H2), there exists $N$ such that

$$
\Delta\left(a_{n} \Delta\left(b_{n} \Delta\left(c_{n} \Delta y_{n}\right)\right)\right)<0, \quad \text { for } n \geq N \text {. }
$$

Summing the above inequality form $N$ to $n-1$, we get

$$
\Delta\left(b_{n} \Delta\left(c_{n} \Delta y_{n}\right)\right)<\frac{A_{0}}{a_{n}}, \quad \text { for } n \geq N+1,
$$

where $A_{0}$ is a constant.

Summing again, we have

$$
b_{n} \Delta\left(c_{n} \Delta y\right)<A_{0} \sum_{i=N}^{n-1} \frac{1}{a_{i}}+b_{N} \Delta\left(c_{N} \Delta y_{N}\right) \text {, }
$$

and therefore,

$$
\Delta\left(c_{n} \Delta y_{n}\right)<\frac{A_{0}}{b_{n}} \sum_{i=N}^{n-1} \frac{1}{a_{i}}+\frac{A_{1}}{b_{n}}, \quad \text { for } n \geq N+1 .
$$

Summing the last inequality, we obtain

$$
c_{n} \Delta y_{n}<A_{0} \sum_{j=N}^{n-1} \frac{1}{b_{j}} \sum_{l=N}^{j-1} \frac{1}{a_{i}}+A_{1} \sum_{j=1}^{n-1} \frac{1}{b_{j}}+A_{2}, \quad n \geq N+2,
$$

where $A_{1}$ and $A_{2}$ are constants. 
Hence,

$$
\Delta y_{n}<\frac{A_{0}}{c_{n}} \sum_{j=N}^{n-1} \frac{1}{b_{j}} \sum_{i=N}^{j-1} \frac{1}{a_{i}}+\frac{A_{1}}{c_{n}} \sum_{j=1}^{n-1} \frac{1}{b_{j}}+\frac{A_{2}}{c_{n}} .
$$

A final summation yields

$$
y_{n}<A_{0} \sum_{k=N}^{n-1} \frac{1}{c_{k}} \sum_{j=N}^{k} \frac{1}{b_{j}} \sum_{i=N}^{j-1} \frac{1}{a_{i}}+A_{1} \sum_{k=N}^{n-1} \frac{1}{c_{k}} \sum_{j=N}^{k-1} \frac{1}{b_{k}}+A_{2} \sum_{k=N}^{n-1} \frac{1}{c_{k}}+A_{3}, \quad n \geq N+3 .
$$

It is easy to see that every term on the right-hand side of the last inequality is less than $Q_{n, N}$. Therefore, we obtain $y_{n} \leq C_{2} Q_{n, N}$ for $n \geq N+3$, where $C_{2}$ is a positive constant.

We say that a nonoscillatory solution $\left(y_{n}\right)$ of (1.1) is asymptotically constant if there exist some positive constant $\alpha$ such that $y_{n} \rightarrow \alpha$ and asymptotically $Q_{n, N}$ if there is some positive constant $\beta$ such that $y_{n} / Q_{n, N} \rightarrow \beta$.

According to Lemma 2.5, we may regard an asymptotically constant solution as a "minimal" solution, and an asymptotically $Q_{n, N}$ solution as a "maximal" solution.

Now, we present a necessary and sufficient condition for the existence of an asymptotically $Q_{n, N}$ solution.

Theorem 2.6. Assume that (H1), (H2), and (H3) hold and $f$ is a nondecreasing function in another argument, that is, " $f(n, t 1) \leq f(n, t 2)$ for $t 1<t 2$ and each fixed $n$." Then a necessary and sufficient condition for (1.1) to have a solution $\left(y_{n}\right)$ satisfying

$$
\lim _{n \rightarrow \infty} \frac{y_{n}}{Q_{n, N}}=\beta \neq 0
$$

is that

$$
\sum_{n=1}^{\infty}\left|f\left(n, C Q_{n, N}\right)\right|<\infty
$$

for some integer $N \geq 1$ and some nonzero constant $C$.

Proof

Necessity. Let $\left(y_{n}\right)$ be a nonoscillatory solution of (1.1) which satisfies (2.29). Without loss of generality, we may assume that $\beta>0$. Then there exist positive numbers $d_{1}$ and $d_{2}$ such that

$$
d_{1} Q_{n, N} \leq y_{n} \leq d_{2} Q_{n, N}, \quad n \geq N+3
$$

where $N$ is a sufficiently large integer. Then

$$
f\left(n, y_{n}\right) \geq f\left(n, d_{1} Q_{n}\right) .
$$


On the other hand, summing (1.1) from $N$ to $n-1$, and from Lemma 1.1, we get

$$
0<a_{n} \Delta\left(b_{n} \Delta\left(c_{n} \Delta y_{n}\right)\right)=a_{N} \Delta\left(b_{N} \Delta\left(c_{N} \Delta y_{N}\right)\right)-\sum_{i=N}^{n-1} f\left(i, y_{i}\right),
$$

which implies that

$$
\sum_{i=N}^{\infty} f\left(i, y_{i}\right) \leq a_{N} \Delta\left(b_{N} \Delta\left(c_{N} \Delta y_{N}\right)\right)<\infty
$$

So, by (2.32), we have

$$
\sum_{i=N}^{\infty} f\left(i, d_{1} Q_{i, N}\right)<\infty
$$

Sufficiency. Assume that (2.30) holds with $C>0$ since a similar argument holds if $C<0$. Let $N$ be large enough that

$$
\sum_{i=N-3}^{\infty} f\left(i, C Q_{n, N}\right)<\frac{1}{8} C
$$

Consider the Banach space $B_{N}$ of all real sequences $y=\left(y_{n}\right)$ defined for $n \geq N+3$ such that

$$
\|y\|=\sup _{n \geq N+3} \frac{\left|y_{n}\right|}{Q_{n, N}^{2}}<\infty .
$$

Let $S$ be the subset of $B_{N}$ defined by

$$
S=\left\{\left(y_{n}\right) \in B_{N}: \frac{C}{2} Q_{n, N} \leq y_{n} \leq C Q_{n, N}, n \geq N+3\right\} .
$$

It is not difficult to see that $S$ is a bounded, convex, and closed subset of $B_{N}$.

We define a mapping $T: S \rightarrow B_{N}$ as follows:

$$
\begin{aligned}
(T y)_{n}= & \frac{C}{2} Q_{n, N}+Q_{n, N} \sum_{i=n-1}^{\infty} F(i)+\sum_{j=N}^{n-1} F(j-1) Q_{j, N} \\
& +\sum_{i=N}^{n-1} \frac{1}{c_{i}} \sum_{j=N}^{i-1} F(j) \sum_{k=N}^{j-1} \frac{1}{b_{k}} \sum_{s=N}^{k-1} \frac{1}{a_{s}} \\
& +\sum_{i=N}^{n-1} \frac{1}{c_{i}} \sum_{j=N}^{i-1} \frac{1}{b_{j}} \sum_{k=N}^{j-1} F(k+1) \sum_{s=N}^{k-1} \frac{1}{a_{s}}, \quad \text { for } n \geq N+3,
\end{aligned}
$$

where we have used the notation $F(k)$ for denoting $f\left(k-2, y_{(k-2)}\right)$. 
We first show that $T(S) \subset S$. Indeed, if $y \in S$, it is clear from (2.39) that $(T y)_{n} \geq$ $(C / 2) Q_{n, N}$ for $n \geq N+3$. Furthermore, for $n \geq N+3$, we have

$$
\begin{aligned}
(T y)_{n} \leq & \frac{C}{2} Q_{n, N}+Q_{n, N} \sum_{i=n-1}^{\infty} F(i)+Q_{n, N} \sum_{j=N}^{n-1} F(j-1) \\
& +\sum_{i=N}^{n-1} \frac{1}{c_{i}} \sum_{k=N}^{i-1} \frac{1}{b_{k}} \sum_{s=N}^{k-1} \frac{1}{a_{s}} \sum_{j=k+1}^{i-1} F(j) \\
& +\sum_{i=N}^{n-1} \frac{1}{c_{i}} \sum_{j=N}^{i-1} \frac{1}{b_{j}} \sum_{s=N}^{j-1} \frac{1}{a_{s}} \sum_{k=s+1}^{j-1} F(k+1) \\
\leq & \frac{C}{2} Q_{n, N}+Q_{n, N} \sum_{i=N-3}^{\infty} F(i+2)+Q_{n, N} \sum_{j=N-3}^{\infty} F(j+2) \\
& +Q_{n, N} \sum_{j=N-1}^{\infty} F(j+2)+Q_{n, N} \sum_{k=N}^{\infty} F(k+2) \\
\leq & \frac{C}{2} Q_{n, N}+4 Q_{n, N} \sum_{i=N-3}^{\infty} F(i+2) .
\end{aligned}
$$

So, we have

$$
(T y)_{n} \leq \frac{C}{2} Q_{n, N}+4 Q_{n, N} \sum_{i=N-3}^{\infty} f\left(i, y_{i}\right) .
$$

Therefore, by (2.36), we get

$$
(T y)_{n} \leq \frac{C}{2} Q_{n, N}+4 Q_{n, N} \sum_{i=N-3}^{\infty} f\left(i, C Q_{i, N}\right) \leq C Q_{n, N} .
$$

Thus $T$ maps $S$ into itself.

Next we prove that $T$ is continuous. Let $\left(y^{(m)}\right)$ be a sequence in $S$ such that $y^{(m)} \rightarrow y$ as $m \rightarrow \infty$. Because $S$ is closed, $y \in S$. Now, by (2.41), we get

$$
\left|\left(T y^{(m)}\right)_{n}-(T y)_{n}\right| \leq 4 Q_{n, N} \sum_{i=N-3}^{\infty}\left|f\left(i, y_{i}^{(m)}\right)-f\left(i, y_{i}\right)\right|, \quad n \geq N+3
$$

and therefore,

$$
\left\|\left(T y^{(m)}\right)_{n}-(T y)_{n}\right\| \leq \frac{4}{Q_{n, N}} \sum_{i=N-3}^{\infty}\left|f\left(i, y_{i}^{(m)}\right)-f\left(i, y_{i}\right)\right| .
$$


Since

$$
\begin{gathered}
\lim _{m \rightarrow \infty}\left|f\left(i, y_{i}^{(m)}\right)-f\left(i, y_{i}\right)\right|=0, \\
\left|f\left(i, y_{i}^{(m)}\right)-f\left(i, y_{i}\right)\right| \leq 2 f\left(i, C Q_{i, N}\right), \quad \text { for } i \geq N+3,
\end{gathered}
$$

we see from Lebesgue's dominated convergence theorem that

$$
\lim _{m \rightarrow \infty}\left\|T y^{(m)}-T y\right\|=0 .
$$

This means that $T$ is continuous.

Finally, we need to show that $T(S)$ is uniformly Cauchy. To see this, we have to show that, given any $\epsilon>0$, there exists an integer $N_{1}$ such that, for $m>n>N_{1}$,

$$
\left|\frac{(T y)_{m}}{Q_{m, N}^{2}}-\frac{(T y)_{n}}{Q_{n, N}^{2}}\right|<\epsilon,
$$

for any $y \in S$. Indeed, by (2.41) and (2.36), we have

$$
\left|\frac{(T y)_{m}}{Q_{m, N}^{2}}-\frac{(T y)_{n}}{Q_{n, N}^{2}}\right| \leq \frac{C}{Q_{n, N}}+\frac{8}{Q_{n, N}} \sum_{i=N-3}^{\infty} f\left(i, y_{i}\right) \leq \frac{2 C}{Q_{n, N}} \longrightarrow 0
$$

Therefore, by Lemma 1.1, there exists $y \in S$ such that $y_{n}=(T y)_{n}$, for $n \geq N+3$. It is easy to see that $\left(y_{n}\right)$ is a solution of (1.1). Furthermore, by Stolz's theorem, we have

$$
\begin{aligned}
\lim _{n \rightarrow \infty} \frac{y_{n}}{Q_{n, N}} & =\lim _{n \rightarrow \infty} \frac{\Delta y_{n}}{\Delta Q_{n, N}}=\lim _{n \rightarrow \infty} \frac{c_{n} \Delta y_{n}}{c_{n} \Delta Q_{n, N}}=\lim _{n \rightarrow \infty} \frac{\Delta\left(c_{n} \Delta y_{n}\right)}{\Delta\left(c_{n} \Delta Q_{n, N}\right)} \\
& =\lim _{n \rightarrow \infty} \frac{b_{n} \Delta\left(c_{n} \Delta y_{n}\right)}{b_{n} \Delta\left(c_{n} \Delta Q_{n, N}\right)}=\lim _{n \rightarrow \infty} \frac{\Delta\left(b_{n} \Delta\left(c_{n} \Delta y_{n}\right)\right)}{\Delta\left(\sum_{i=1}^{n-1}\left(1 / a_{i}\right)\right)} \\
& =\lim _{n \rightarrow \infty} a_{n} \Delta\left(b_{n} \Delta\left(c_{n} \Delta y_{n}\right)\right),
\end{aligned}
$$

so,

$$
\lim _{n \rightarrow \infty} \frac{y_{n}}{Q_{n, N}}=\lim _{n \rightarrow \infty}\left(C+\sum_{s=n+2}^{\infty} G(s)\right)=\lim _{n \rightarrow \infty}\left(C+\sum_{s=n+2}^{\infty} f\left(i, y_{i}\right)\right)=C .
$$

This completes the proof.

Theorem 2.6 extends [13, Theorem 1] and [14, Theorem 3].

Example 2.7. Consider the difference equation

$$
\Delta\left(\frac{1}{n} \Delta\left((n-1) \Delta\left((n-1) \Delta y_{n}\right)\right)\right)+\frac{1}{n^{5 / 3}(n+1)} y_{n}^{1 / 3}=0, \quad \text { for } n \geq 2 .
$$


It is easy to calculate that $Q_{n, N}=(1 / 8) n(n+1), n \geq 4$. Hence the above equation has a solution $\left(y_{n}\right)$ such that $\lim _{n \rightarrow \infty}\left(y_{n} / Q_{n, N}\right)=C \neq 0$. In fact, $y_{n}=n^{2}$ is a solution of this equation with $\lim _{n \rightarrow \infty}\left(y_{n} / Q_{n, N}\right)=8$.

Next we derive a necessary and sufficient condition for the existence of an asymptotically constant solution of (1.1).

Theorem 2.8. Assume that (H1), (H2), and (H3) hold and the function $f$ is a monotonic function in the second argument. Then a necessary and sufficient condition for (1.1) to have a solution $\left(y_{n}\right)$ which satisfies

$$
\lim _{n \rightarrow \infty} y_{n}=\alpha \neq 0
$$

is that

$$
\sum_{i=1}^{\infty} P_{i, N}|f(i, c)|<\infty,
$$

for some integer $N \geq 1$ and some nonzero constant $c$.

Proof

Necessity. Without loss of generality, we assume that $\left(y_{n}\right)$ is an eventually positive solution of (1.1) such that

$$
\lim _{n \rightarrow \infty} y_{n}=\alpha>0
$$

Then there exist positive constants $d_{3}$ and $d_{4}$ such that

$$
d_{3} \leq y_{n} \leq d_{4}, \quad \text { for large } n \text {. }
$$

Let $z_{n}=b_{n} \Delta\left(c_{n} \Delta y_{n}\right)$. It is clear that if condition ( $\left.\mathrm{H} 1\right)$ is satisfied, then solution $\left(y_{n}\right)$ of (1.1) of type (i) tends to infinity. Since $\left(y_{n}\right)$ satisfies condition (ii) of Lemma 2.1, hence $y_{n}>0, z_{n}<0, \Delta y_{n}>0$, and $\Delta z_{n}>0$ eventually. Let $N$ be so large that (2.55) and (ii) hold for $n \geq N$. We will use (1.1) in the following form:

$$
\Delta\left(a_{n-2} \Delta z_{n-2}\right)=-f\left(n-2, y_{(n-2)}\right) .
$$

Multiplying the above equation by $P_{i-2, N-2}$, and summing both sides of it from $i=N$ to $n-2$, we obtain

$$
\sum_{i=N}^{n-2} P_{i-2, N-2} f\left(i-2, y_{(i-2)}\right)=-\sum_{i=N}^{n-2} P_{i-2, N-2} \Delta\left(a_{i-2} \Delta z_{i-2}\right) .
$$


Hence, by the formula $\sum_{i=K}^{n-2} y_{i} \Delta x_{i}=\left.x_{i} y_{i}\right|_{i=K} ^{n-1}-\sum_{i=K}^{n-2} x_{i+1} \Delta y_{i}$, we get

$$
\begin{aligned}
\sum_{i=N}^{n-2} P_{i-2, N-2} f\left(i-2, y_{(i-2)}\right) & \\
= & -\left.P_{i-2, N-2} a_{i-2} \Delta z_{i-2}\right|_{i=N} ^{n-1}+\sum_{i=N}^{n-2}\left(\Delta P_{i-2, N-2}\right) a_{i-1} \Delta z_{i-1} \\
= & -P_{n-3, N-2} a_{n-3} \Delta z_{n-3}+\sum_{i=N}^{n-2} \frac{1}{a_{i-1}} \sum_{j=N}^{i-1} \frac{1}{b_{j}} \sum_{k=N}^{j} \frac{1}{c_{k}} a_{i-1} \Delta z_{i-1} \\
< & \sum_{i=N}^{n-2}\left(\sum_{j=N}^{i-1} \frac{1}{b_{j}} \sum_{k=N}^{j} \frac{1}{c_{k}}\right) \Delta z_{i-1} \\
= & \left.\sum_{j=N}^{i-1} \frac{1}{b_{j}} \sum_{k=N}^{j} \frac{1}{c_{k}} z_{i-1}\right|_{i=N} ^{n-1}-\sum_{i=N}^{n-2}\left(\frac{1}{b_{i}} \sum_{j=N}^{i} \frac{1}{c_{j}}\right) \Delta z_{i} \\
= & \sum_{j=N}^{n-2} \frac{1}{b_{j}} \sum_{k=N}^{j} \frac{1}{c_{k}} z_{n-1}-\sum_{i=N}^{n-2} \frac{1}{b_{i}} \sum_{j=N}^{i} \frac{1}{c_{j}} b_{i} \Delta\left(c_{i} \Delta y_{i}\right) \\
< & -\sum_{i=N}^{n-2}\left(\sum_{j=N}^{i} \frac{1}{c_{j}}\right) \Delta\left(c_{i} \Delta y_{i}\right) \\
= & -\sum_{j=N}^{n-1} \frac{1}{c_{j}}\left(y_{N}+y_{n}-y_{N+1} \Delta y_{n-1}\right)+\Delta y_{N}+\Delta y_{n-1}+\cdots+\Delta y_{N+1}+y_{n} ; \\
= & \left.\frac{1}{c_{j}}\left(c_{i} \Delta y_{i}\right)\right|_{i=N} ^{n-1}+\sum_{i=N}^{n-2} \Delta y_{i+1} \\
&
\end{aligned}
$$

which tends to $\Delta y_{N}+\alpha$ as $n \rightarrow \infty$. Therefore,

$$
\sum_{i=N}^{\infty} P_{i-2, N-2} f\left(i-2, y_{(i-2)}\right)<\infty .
$$

From the monotonicity of the function $f$, we get

$$
f\left(i-2, y_{(i-2)}\right) \geq f\left(i-2, d_{3}\right)
$$

when $f$ is nondecreasing and

$$
f\left(i-2, y_{(i-2)}\right) \geq f\left(i-2, d_{4}\right)
$$

when $f$ is nonincreasing. This implies that

$$
\sum_{i=1}^{\infty} P_{i-2, N-2} f\left(i-2, d_{k}\right)<\infty,
$$


where $k=3$ for $f$ nondecreasing and $k=4$ for $f$ nonincreasing. Then the necessity of this theorem holds.

Sufficiency. Next, let $M \geq 1$ be a large integer such that, for some nonzero constant $c$, $\sum_{i=M}^{\infty} P_{i, M} f(i, c)<\alpha / 4$. From here,

$$
\sum_{i=M+2}^{\infty} \frac{1}{c_{i}} \sum_{j=i}^{\infty} \frac{1}{b_{j}} \sum_{k=j}^{\infty} \frac{1}{a_{k}} \sum_{s=k}^{\infty} f(s, c)<\frac{\alpha}{4} .
$$

Here $\alpha=\beta / 2$ if $f$ is nondecreasing and $\alpha=\beta$ when $f$ is nonincreasing. Let $B$ be the linear space of a bounded real sequences $\left(y_{n}\right)_{N}^{\infty}$ with the usual operations and supremum norm. Let $S$ be the subset

$$
S=\left\{\left(y_{n}\right) \in B ; \alpha \leq y_{n} \leq 2 \alpha, n \geq M+3\right\} .
$$

It is easy to see that $S$ is a bounded, convex, and closed subset of $B$.

We define operator $T$ in the following way:

$$
\begin{aligned}
(T y)_{n}= & \alpha+\left(\sum_{s=M+2}^{n-1} \frac{1}{c_{s}} \sum_{k=n}^{\infty} \frac{1}{b_{k}} \sum_{j=k}^{\infty} \frac{1}{a_{j}} \sum_{i=j}^{\infty} f\left(i, y_{i}\right)\right) \\
& +\left(\sum_{k=M+2}^{n-1} \frac{1}{b_{k}} \sum_{s=M+2}^{k} \frac{1}{c_{s}}\right) \sum_{j=n}^{\infty} \frac{1}{a_{j}} \sum_{i=j}^{\infty} f\left(i, y_{i}\right) \\
& +\left(\sum_{j=M+2}^{n-1} \frac{1}{a_{j}} \sum_{k=M+2}^{j} \frac{1}{b_{k}} \sum_{s=M+2}^{k} \frac{1}{c_{s}}\right) \sum_{i=n}^{\infty} f\left(i, y_{i}\right) \\
& +\sum_{i=M+2}^{n-1}\left[\left(\sum_{j=M+2}^{i} \frac{1}{a_{j}} \sum_{k=M+2}^{j} \frac{1}{b_{k}} \sum_{s=M+2}^{k} \frac{1}{c_{s}}\right) f\left(i, y_{i}\right)\right],
\end{aligned}
$$

for $n \geq M+3$. It is easy to see that $T$ maps $S$ into itself. Indeed, if $y_{n} \in S$, then $(T y)_{n} \geq \alpha$. Furthermore, for $n \geq M+3$, we have

$$
\begin{aligned}
\left(\sum_{s=M+2}^{n-1} \frac{1}{c_{s}}\right) \sum_{k=n}^{\infty} \frac{1}{b_{k}} \sum_{j=k}^{\infty} \frac{1}{a_{j}} \sum_{i=j}^{\infty} f\left(i, y_{i}\right) & \leq \sum_{s=M+2}^{\infty} \frac{1}{c_{s}} \sum_{k=n}^{\infty} \frac{1}{b_{k}} \sum_{j=k}^{\infty} \frac{1}{a_{j}} \sum_{i=j}^{\infty} f\left(i, y_{i}\right) \\
& \leq \sum_{s=M+2}^{\infty} \frac{1}{c_{s}} \sum_{k=s}^{\infty} \frac{1}{b_{k}} \sum_{j=k}^{\infty} \frac{1}{a_{j}} \sum_{i=j}^{\infty} f\left(i, y_{i}\right)<\frac{\alpha}{4}, \\
\left(\sum_{k=M+2}^{n-1} \frac{1}{b_{k}} \sum_{s=M+2}^{k} \frac{1}{c_{s}}\right) \sum_{j=n}^{\infty} \frac{1}{a_{j}} \sum_{i=j}^{\infty} f\left(i, y_{i}\right) & \leq \sum_{s=M+2}^{n-1} \frac{1}{c_{s}} \sum_{k=s}^{n-1} \frac{1}{b_{k}} \sum_{j=n}^{\infty} \frac{1}{a_{j}} \sum_{i=j}^{\infty} f\left(i, y_{i}\right) \\
& \leq \sum_{s=M+2}^{n-1} \frac{1}{c_{s}} \sum_{k=s}^{n-1} \frac{1}{b_{k}} \sum_{j=k}^{\infty} \frac{1}{a_{j}} \sum_{i=j}^{\infty} f\left(i, y_{i}\right)<\frac{\alpha}{4}
\end{aligned}
$$

It follows that $(T y)_{n}<\alpha / 4+\alpha / 4+\alpha / 4+\alpha / 4+\alpha=2 \alpha$. 
Next we assert that $T$ is continuous. Let $y^{(m)} \rightarrow y$ as $m \rightarrow \infty$. We derive that

$$
(T y)_{n}<\alpha+4 \sum_{s=M+2}^{\infty} \frac{1}{c_{s}} \sum_{k=s}^{\infty} \frac{1}{b_{k}} \sum_{j=k}^{\infty} \frac{1}{a_{j}} \sum_{i=j}^{\infty} f\left(i, y_{i}\right)
$$

for $n \geq M+3$.

We obtain

$$
\begin{aligned}
\left|\left(T y^{(m)}\right)_{n}-(T y)_{n}\right|<4 & \mid \sum_{s=M+2}^{\infty} \frac{1}{c_{s}} \sum_{k=s}^{\infty} \frac{1}{b_{k}} \sum_{j=k}^{\infty} \frac{1}{a_{j}} \sum_{i=j}^{\infty} f\left(i, y_{i}^{(m)}\right) \\
& -\sum_{s=M+2}^{\infty} \frac{1}{c_{s}} \sum_{k=s}^{\infty} \frac{1}{b_{k}} \sum_{j=k}^{\infty} \frac{1}{a_{j}} \sum_{i=j}^{\infty} f\left(i, y_{n}\right) \mid \\
& <4 \sum_{s=M+2}^{\infty} \frac{1}{c_{s}} \sum_{k=s}^{\infty} \frac{1}{b_{k}} \sum_{j=k}^{\infty} \frac{1}{a_{j}} \sum_{i=j}^{\infty}\left|f\left(i, y_{i}^{(m)}\right)-f\left(i, y_{i}\right)\right| .
\end{aligned}
$$

Hence,

$$
\left\|T y^{(m)}-T y\right\| \leq 4 \sum_{s=M+2}^{\infty} \frac{1}{c_{s}} \sum_{k=s}^{\infty} \frac{1}{b_{k}} \sum_{j=k}^{\infty} \frac{1}{a_{j}} \sum_{i=j}^{\infty}\left|f\left(i, y_{i}^{(m)}\right)-f\left(i, y_{i}\right)\right| .
$$

Since

$$
\begin{gathered}
\lim _{m \rightarrow \infty}\left|f\left(i, y_{i}^{(m)}\right)-f\left(i, y_{i}\right)\right|=0, \\
\left|f\left(i, y_{i}^{(m)}\right)-f\left(i, y_{i}\right)\right| \leq 2 f(i, C),
\end{gathered}
$$

then, from Lebesgue's dominated convergence theorem, we see that

$$
\lim _{m \rightarrow \infty}\left\|T y^{(m)}-T y\right\|=0 .
$$

This proves our assertion.

It is easy to see that $T(S)$ is uniformly Cauchy.

Thus, by Lemma 1.1, the operator $T$ has a fixed point in $S$. One can see that $\left(y_{n}\right)$ is a solution of $(1.1)$ for all large $n$, then $\lim _{n \rightarrow \infty} y_{n} \in[\alpha, 2 \alpha]$ as required.

Theorem 2.8 extends [13, Theorem 2] and [14, Theorem 4].

Example 2.9. Consider the difference equation

$$
\Delta\left(n \Delta\left((n-1) \Delta\left(\frac{1}{(n-1)} \Delta y_{n}\right)\right)\right)+\frac{27 n^{3}}{(n+4)(n+3)(n+2)(n+1)(n-1)^{3}} y_{n}{ }^{3}=0,
$$

for $n \geq 2$. All assumptions of Theorem 2.8 are satisfied. Therefore, the above equation has solution $\left(y_{n}\right)$ such that $\lim _{n \rightarrow \infty} y_{n}=\alpha \neq 0$. In fact, $y_{n}=1-1 / n$ is such a solution. 
In conclusion, note that Theorems 2.6 and 2.8 can be easily extended to equations of the form

$$
\Delta\left(a_{n} \Delta\left(b_{n} \Delta\left(c_{n} \Delta y_{n}\right)\right)\right)+f\left(n, y_{\delta_{n}}\right)=0, \quad n \in \mathbb{N},
$$

where $\delta$ is an integer-valued function defined on $\mathbb{N}$ such that $\lim _{n \rightarrow \infty} \delta_{n}=\infty$.

\section{References}

[1] S. S. Cheng, On a class of fourth-order linear recurrence equations, Int. J. Math. Math. Sci. 7 (1984), no. 1, 131-149.

[2] J. R. Graef and E. Thandapani, Oscillatory and asymptotic behavior of fourth order nonlinear delay difference equations, Fasc. Math. (2001), no. 31, 23-36.

[3] J. W. Hooker and W. T. Patula, Growth and oscillation properties of solutions of a fourth order linear difference equation, J. Austral. Math. Soc. Ser. B 26 (1985), no. 3, 310-328.

[4] J. Popenda and E. Schmeidel, On the solutions of fourth order difference equations, Rocky Mountain J. Math. 25 (1995), no. 4, 1485-1499.

[5] E. Schmeidel, Nonscillation and oscillation properties for fourth order nonlinear difference equations, New Progress in Difference Equations (B. Aulbach, S. Elaydi, and G. Ladas, eds.), Taylor \& Francis, London, 529-536, in press.

[6] - Oscillation and nonoscillation theorems for fourth order difference equations, Rocky Mountain J. Math. 33 (2003), no. 3, 1083-1094.

[7] E. Schmeidel and B. Szmanda, Oscillatory and asymptotic behavior of certain difference equation, Nonlinear Anal. 47 (2001), no. 7, 4731-4742.

[8] B. Smith and W. E. Taylor Jr., Oscillatory and asymptotic behavior of certain fourth order difference equations, Rocky Mountain J. Math. 16 (1986), no. 2, 403-406.

[9] _ Oscillation properties of fourth order linear difference equations, Tamkang J. Math. 18 (1987), no. 4, 89-95.

[10] W. E. Taylor Jr., Oscillation properties of fourth order difference equations, Portugal. Math. 45 (1988), no. 1, 105-114.

[11] W. E. Taylor Jr. and M. Sun, Oscillation properties of nonlinear difference equations, Portugal. Math. 52 (1995), no. 1, 15-24.

[12] E. Thandapani and I. M. Arockiasamy, Oscillatory and asymptotic properties of solutions of nonlinear fourth order difference equations, Glas. Mat. Ser. III 37(57) (2002), no. 1, 119-131.

[13] J. Yan and B. Liu, Oscillatory and asymptotic behaviour of fourth order nonlinear difference equations, Acta Math. Sinica (N.S.) 13 (1997), no. 1, 105-115.

[14] B. G. Zhang and S. S. Cheng, On a class of nonlinear difference equations, J. Differ. Equations Appl. 1 (1995), no. 4, 391-411.

Małgorzata Migda: Institute of Mathematics, Poznań University of Technology, ul. Piotrowo 3a, 60-695 Poznań, Poland

E-mail address: mmigda@math.put.poznan.pl

Anna Musielak: Institute of Mathematics, Poznań University of Technology, ul. Piotrowo 3a, 60-695 Poznań, Poland

E-mail address: musielak@math.put.poznan.pl

Ewa Schmeidel: Institute of Mathematics, Poznań University of Technology, ul. Piotrowo 3a, 60-695 Poznań, Poland

E-mail address: eschmeid@math.put.poznan.pl 